\title{
A 71 MEGAPIXEL MOSAIC CAMERA FOR WEAK LENSING AT APO
}

\author{
A. DIERCKS, C. STUBBS, C. HOGAN AND E. ADELBERGER \\ Departments of Physics and Astronomy \\ University of Washington \\ Box 351580 \\ Seattle, Washington 98195-1580
}

\begin{abstract}
We are developing a wide-field CCD camera system which is optimized for using weak gravitational lensing to study the distribution of dark matter in clusters of galaxies and eventually the field. The system will be used at the Apache Point Observatory (APO) 3.5 meter telescope in New Mexico.
\end{abstract}

\section{Introduction}

One of the significant difficulties with weak gravitational lensing studies is the need for high-resolution, wide-field imaging instruments. The camera described here is designed to meet this challenge by imaging a 30 arcminute diameter field of view with distortion free optics. The system is optimized for TDI (drift scan) operation but will also be capable of taking staring mode images. We have determined that it is most cost effective to use a simple distortion free corrector at the native plate scale of the telescope (f/10) and use a large number of CCDs to cover the entire focal plane.

The camera system contains several novel features which will help meet the demanding imaging requirements of weak gravitational lensing studies. The camera will contain dedicated, fast-readout CCDs for guiding and eventually tip-tilt correction via the secondary mirror of the $3.5 \mathrm{~m}$ telescope as well as a tilted CCD in the focal plane to allow active focusing. The camera, corrector optics, and filters will be able to rotate with respect to each other and the telescope optics in order to study and eliminate systematic effects due to any aberrations in the optical chain. 


\section{Observational Technique}

Our observational approach is driven by the need for measurements that are designed to suppress sources of systematic error. Telescope guiding effects, pixellation effects, optical aberrations, and the effects of seeing need to be properly characterized and suppressed in order to avoid spurious coherent shape distortions in the images. This involves the use of stellar PSF's and so it is imperative that the weak lensing program have well sampled stellar PSF's. Also, large numbers of galaxies need to be observed in multiple passbands in order to gain the statistical leverage necessary to disentangle a weak lensing system.

We will take very deep images of blank and cluster fields with the redundancy that is imperative if a weak lensing signal is to be distinguished from systematic effects. The target galaxies have surface brightnesses of 27 th mag $(\mathrm{R})$ per square arcsec, so they are well below $1 \%$ of sky. We intend to (1) rotate the corrector plus instrument to several position angles on the sky, (2) take two full independent sets of data for each field, (3) take images in three passbands for redundancy and color discrimination, (4) rotate the CCD camera, corrector optics, filters, and telescope with respect to each other, and (5) operate in both drift scan and staring mode, as these two techniques have different sensitivities to a number of the sources of systematic error that concern us. We have endeavored to create a design where it is easy to perform these operations as rapidly as possible.

By demanding that the observed ellipticity distribution of the galaxies be invariant under these various operations we hope to suppress the majority of telescope and instrument artifacts that could produce a false signal, or mask a real one.

\section{The APO $3.5 \mathrm{~m}$ Telescope}

The camera system is engineered to take advantage of the unique capabilities of the APO $3.5 \mathrm{~m}$ telescope. One of these features is the ability to track at any rate and direction on the sky. Thus it is possible to "drift scan" at an arbitrary rate in an arbitrary direction, and thereby obtain long integrations. We have already employed this feature to great advantage using a smaller camera system currently available on the telescope. In drift scan mode we will be able to image a swath nearly 30 arcminutes wide, twice on a single pass. Alternatively, we will have the option of imaging the swath simultaneously in two colors. 